\title{
Perspectives on Cutting-Edge Clinical Trials
}

\section{A Conversation Between Michael Hofman, Johannes Czernin, and Jérémie Calais}

\author{
Michael S. Hofman ${ }^{1}$, Jérémie Calais ${ }^{2}$, and Johannes Czernin ${ }^{2}$ \\ ${ }^{1}$ Peter MacCallum Cancer Centre, Melbourne, Victoria, Australia; and ${ }^{2}$ Ahmanson Translational Theranostics Division, Department of \\ Molecular \& Medical Pharmacology, David Geffen School of Medicine, University of California Los Angeles, Los Angeles, California
}

$\mathbf{J}$ ohannes Czernin, MD, editor in chief of The Journal of Nuclear Medicine, and Jérémie Calais, MD, MSc, his colleague at UCLA, talked with Michael Hofman, MBSS, about success and innovation in clinical trial development. Hofman is a nuclear medicine physician at the Peter MacCallum Cancer Centre (Melbourne), Australia's only public hospital dedicated to cancer treatment, research, and education. He has a coappointment at the University of Melbourne and previously completed a fellowship at Guy's and St. Thomas's hospital in London (U.K.). He is the director of the Prostate Cancer Theranostics and Imaging Centre of Excellence at Peter Mac. His research focuses on improving outcomes in prostate cancer, and he is engaged in both preclinical and phases 1-3 research. He has led several landmark clinical trials of prostate-specific membrane antigen (PSMA) imaging and therapy, including the ProPSMA (PSMA PET/CT in patients with high-risk cancer before curativeintent surgery or radiotherapy) study, which has established PSMA PET/CT as a replacement for standard CT and bone scanning, and the TheraP $\left({ }^{177} \mathrm{Lu}-\mathrm{PSMA} 617\right.$ theranostic vs. cabazitaxel in progressive metastatic castration-resistant prostate cancer) study. He has authored or coauthored more than 200 peer-reviewed articles and is a scientific member of the Australasian Radiopharmaceutical Trials Network (ARTnet) and a board member of the SNMMI Theranostic Center of Excellence. In these roles, he aims to advance the field through clinical trial development and execution. He is an editor for several international journals, including The Journal of Nuclear Medicine.

Dr. Czernin: Thank you for participating in today's leadership discussion. You have had great success in designing, executing, and publishing large-scale clinical trials. The latest example is the TheraP trial (1), published in The Lancet earlier this year. We are going to talk about your path to becoming a world-leading imaging and theranostic trial designer.

Dr. Calais: Congratulations on all the great studies you have published. You have become a role model for the younger generation, and your work attracts new trainees to the field. Can you tell us a little bit about your training and how your career in nuclear medicine and theranostics started?

Dr. Hofman: Thanks, Johannes and Jérémie. It's a pleasure and honor to share my journey with you. I initially trained in internal medicine, which is the main pathway to nuclear medicine in Australia, along with radiology. In my training, I cycled through nearly all the medical specialties. I had a background in computer science and was always seeking ways to combine my IT skills and medicine. I

COPYRIGHT $\subset 2021$ by the Society of Nuclear Medicine and Molecular Imaging DOI: 10.2967/jnumed.121.262681 stumbled on nuclear medicine. When I finished my nuclear medicine training in Melbourne, I did a 12-mo clinical research fellowship at Guy's and St. Thomas's in London in 2005 and 2006, exposing me to a high-quality academic environment. When I returned to Melbourne, I worked as an attending physician in a combination of private practice and public hospital environments for 3 years. I really enjoyed it but missed the academic environment. At that time the Peter MacCallum Cancer Centre, led by Rodney Hicks, MD, was looking for an additional faculty member. It was a pioneering department with good infrastructure and a world-leading neuroendocrine tumor program. It was the only department in Australia performing ${ }^{68} \mathrm{Ga}-$ DOTATATE PET imaging. I joined and was embedded into the neuroendocrine tumor program and saw its remarkable value, on both the imaging and the therapeutic sides. And I really liked the mix of PET scanning, therapy, and research.

Dr. Calais: How did you become a "trialist"?

Dr. Hofman: I developed a research interest and expertise in lymphoma PET, ${ }^{68} \mathrm{Ga}$ radiopharmaceuticals, and neuroendocrine tumors, and I had some nice publications in these domains. We succeeded in publishing our data in The Journal of Nuclear Medicine, which was a fantastic outcome for me at the time, but I realized it was not changing global practice. In addition, when I went to oncology conferences, the audience was skeptical about the quality of data that we were producing in nuclear medicine. One of my favorite papers that we authored cycled through submission to 4 highimpact oncology journals, who did not even send the paper out for peer review. Responses were along the lines of "Thank you, Dr. Hofman. This is very nice data, but it's limited by its single center and retrospective nature. Please, consider doing this prospectively and resubmitting your data." I reflected on the range of bias inherent in retrospective versus prospective research.

I saw my first ${ }^{99 \mathrm{~m}} \mathrm{Tc}$ - and ${ }^{68} \mathrm{Ga}$-PSMA images at the European Association of Nuclear Medicine meeting in Birmingham in 2011. The extraordinary tumor contrast resonated with me, and I flagged it as my image of the conference and a potential game changer for prostate cancer. Further data in 2012 coming from Germany for imaging and treatment reinforced this. I thought: rather than doing this as an ad hoc imaging test or compassionate access treatment, why not do this as part of well-designed prospective trials? 
My first Lu-PSMA trial at Peter MacCallum Cancer Centre was a collaboration with one of our radiation oncologists, the late John Violet, MD, PhD (2), whose doctorate was in theranostics. In 2014, we wrote a prospective study protocol for ${ }^{177}$ Lu-PSMA-617 therapy together. I am fortunate to be in a large academic hospital, enabling me to collaborate closely with medical oncology and urology colleagues. I started attending the genitourinary oncology multidisciplinary team meetings and very quickly learned a lot about prostate cancer. Understanding the nuances of prostate cancer management was critical to a successful trial. The results of our 30-patient study were presented in 2017 at the European Society of Medical Oncology in front of thousands of oncologists and were published in Lancet Oncology in 2018 (3). We extended the study to 50 patients (4), and I presented those results at the SNMMI meeting, receiving the award for 2018 SNMMI Image of the Year. This demonstrated to me how impactful high-quality research can be for our patients.

Dr. Czernin: After your Peter Mac phase $2{ }^{177}$ Lu-PSMA clinical trial, you progressed to even more impactful, bigger, multicenter randomized studies. How was this Australian clinical trial network set up?

Dr. Hofman: Performing clinical trials is much like a team sport. Many elements are required to be successful, and winning is not easy. In 2014, a group called ARTnet was formed by Andrew M. Scott, MD, and Roslyn Francis, PhD (5). I expressed interest and became a member of the inaugural scientific committee. Our mission was to establish and coordinate multicenter research of novel radiopharmaceuticals. We decided to apply for a grant to do a multicenter study of PSMA PET for staging prostate cancer, which became the ProPSMA trial. We applied for a competitive grant through the Prostate Cancer Foundation of Australia/Movember Foundation, and
PSMA became available only after our trial was designed. With funding for PSMA PET/CT and trial coordination, enthusiastic investigators from nuclear medicine, urology, and radiation oncology quickly joined the team. We hosted site initiation visits, and the group met at conferences. Critical to the success of proPSMA was engagement of the urologists and radiation oncologists who recruited the patients. A favorable regulatory environment for radiopharmaceuticals and a national network of human research ethics committees (HRECs), which recognize approval from the lead center, were additional ingredients for success.

The ProPSMA study was published in The Lancet in 2020 (6) and was the solid foundation of the network that we subsequently used for our theranostic trials. My message to the nuclear medicine community and young investigators is that these types of trials are critical to the future of our specialty. Another benefit of this approach is that we were suddenly upskilling the next generation of nuclear medicine specialists and creating exciting new career opportunities. I am hopeful that out of this growing network we now have more than a dozen nuclear medicine specialists who have potential to design and lead multicenter clinical trials.

Dr. Calais: You now have 2 Lancet papers in the last 2 years. Tell us a little bit more about this success and about the recently published TheraP study. What are the main differences from the imaging journals?

Dr. Hofman: The TheraP trial is a 200-patient randomized trial comparing ${ }^{177}$ Lu-PSMA-617 with cabazitaxel chemotherapy and conducted at 11 sites around Australia, recently published in The Lancet (Fig. 1) (1). In 2016, this was submitted to a grant call from the Australian and New Zealand Urogenital and Prostate Cancer Trials Group (ANZUP), a urooncology cooperative group. We were successful, and ANZUP assisted by turning the concept into

"Nuclear Medicine is one of the most dynamic and rapidly evolving specialties in medicine. My work balance of imaging, therapy, and research and seeing the benefit this has for patient outcomes makes most days inspiring and motivating."

much to our surprise, we were awarded the grant. This provided us with A\$1.5 million to conduct this randomized imaging study.

My time at Guy's \& St. Thomas's being involved in the landmark lymphoma studies led by Profs. Michael O'Doherty, MSc, MD, and Sally Barrington, MSc, MD, and an invitation from you, Dr. Czernin, while at Peter Mac to participate in a randomized trial of $\mathrm{NaF}$ $\mathrm{PET} / \mathrm{CT}$, taught me a lot about clinical trial conduct and the challenges of trial design, execution, and management. I was also involved in a randomized study led by Dr. Hicks of ${ }^{18} \mathrm{~F}$-fluorocholine PET/CT. We have an excellent biostatistics group at Peter Mac, and I worked closely with them, our urology and radiation oncology colleagues, and consumer representatives to design the ProPSMA study. With ARTnet, we standardized ${ }^{68} \mathrm{Ga}$ production across 10 sites. With the grant funding, I hired a dedicated trial coordinator, who was outstanding. All these elements came together for a successful study. At its peak, we randomized 34 patients in 1 month, completing a 300-patient study almost 9 months ahead of schedule.

Dr. Czernin: Did your coworkers and other centers support this immediately, or was that complicated to set up?

Dr. Hofman: Not everybody has the skill set, time, or ambition to design and conduct a phase 3 clinical trial. At the time of ProPSMA design, few sites were even performing PSMA PET/CT. We selected 10 sites that were already performing ${ }^{68} \mathrm{Ga}$-DOTATATE PET/CT and therefore had the infrastructure and expertise required. ${ }^{18} \mathrm{~F}$ - a robust clinical trial. There were many hurdles to overcome, including standardizing radiopharmaceutical production, developing a system for centralized imaging review, and negotiating industry collaboration and ${ }^{177} \mathrm{Lu}$. I believe the success of TheraP was founded in cooperation between nuclear medicine, medical oncology, and radiation oncology. We all sat in the same room, sharing ideas and designing the protocol. This was a winning formula. If designed by nuclear medicine alone, we might have chosen the wrong comparator arm and had a negative study. If designed by medical oncology alone, it would require an industrial-produced ready-to-ship radiopharmaceutical rather than the TheraP model of hospital radiopharmacy production, which was safe and cost-effective for a phase II study. I served as study chair, supported closely by my oncology colleagues, which along with ProPSMA has morphed me into a clinical trialist. We all become a mixture of different things as our expertise in different domains grows.

Compared with submitting to imaging journals, submitting to The Lancet or other high-impact oncology journals requires a different mindset from the outset. These journals require a prospective protocol and predefined statistical analysis plan. They compare the submitted manuscript with these, and if any deviations without justification are noted, the result will be rejection, even if the quality is high. This requires a clarity of goals and planning that is quite different from the retrospective analyses that appear mainly in imaging journals. 


\section{TheraP Clinical Trial Results}

A randomised phase II trial of ${ }^{177} \mathrm{LU}-P S M A-617$ THERANOSTIC versus CABAZITAXEL CHEMOTHERAPY

\section{ANZUP \\ (}
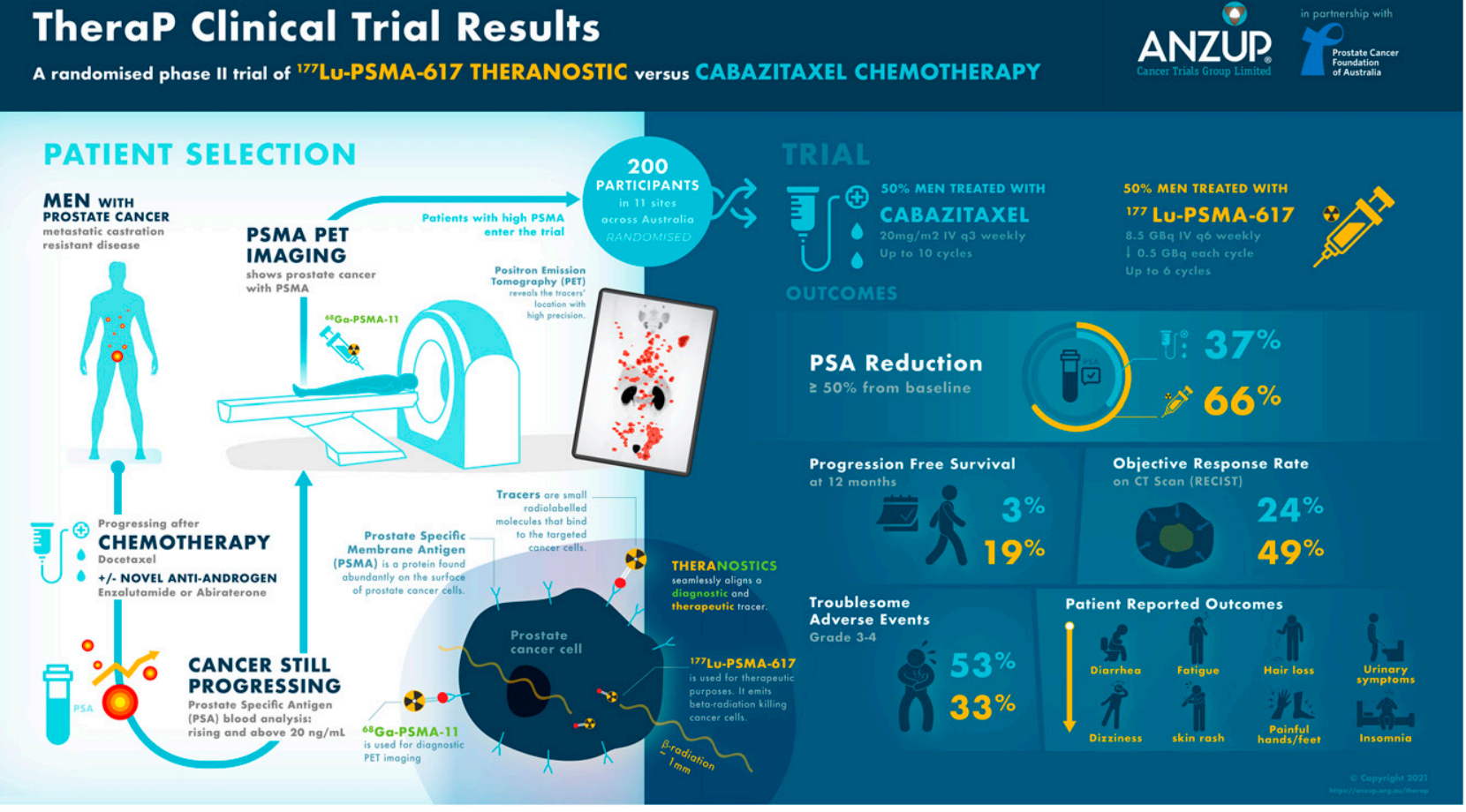

FIGURE 1. Infographic summarizing schema and results of ANZUP TheraP trial.

Dr. Czernin: What is the regulatory environment in Australia? There seems to be a lot of common sense in the ethics committee strategy. What is different from other countries? Is there an Investigational New Drug (IND)-like process?

Dr. Hofman: A huge advantage to conducting nuclear medicine research in Australia is a favorable regulatory environment for validation of novel radiopharmaceuticals. Our U.S. Food and Drug Administration equivalent, the Therapeutic Goods Administration (TGA), has a system that differs from the IND application in the United States. We need to notify TGA that we are doing a trial using an experimental compound, but TGA does not assess or approve the proposal. The TGA essentially defers assessment to institutional HRECs. There are no expensive fees or regulatory approval delays. Once approved by a local HREC, this is recognized by institutions around Australia. In a center such as ours, where the HREC is very familiar with radiopharmaceuticals, it is a relatively smooth pathway, particularly when there are already published data.

Dr. Calais: Nuclear medicine has gained visibility because of the emergence of novel tracers for imaging and theranostics. This makes the field very attractive and interesting, with PSMA being the leading target example. Wall Street is investing a lot of money in companies that own nuclear medicine compounds. Nuclear medicine is at the intersection of radiology, radiation oncology, and medical oncology. Should we adapt our training to better integrate with other specialties? In the United States, there is really a question of who can prescribe radiation treatments such as radioligand therapy or peptide-receptor radionuclide therapy. Who should do what in terms of patient management?

Dr. Hofman: In Australia, most of our current trainees have a radiology background, but we still have a good number of trainees coming from internal medicine. Some people combine 2 medical specialties. Traditionally, these were cardiology and nuclear medicine. Now we have some endocrinology and medical oncology specialists also doing nuclear medicine. I believe there is immense value in having people from different backgrounds. It enriches knowledge in a way that is not possible if everyone comes through exactly the same system. The idea that everyone needs to follow the same training pathway is not the best way to achieve the best outcomes for our patients. Whichever pathway is taken, it is critically important that a good amount of time is spent with direct patient contact before subspecializing. Even for imaging, this is needed to develop the perspective and confidence required to make patientcentered conclusions in our reports rather than merely issuing descriptive reports and devolving decision making to our referrers.

In our theranostics program we are hands-on. We consult patients to evaluate suitability for treatment. This requires a knowledge of other treatment options. We actively contribute to discussions in our multidisciplinary team meetings. We consult patients after treatment, showing patients their responses on PET imaging and discussing optimal follow-up. So we really get to know our patients and interact very closely with our oncology colleagues. We have a dedicated therapy fellow position enabling us to upskill the next generation of practitioners.

Dr. Czernin: Can you comment on the workflow of your theranostics center? Is it a multidisciplinary setup with consulting offices so that all kinds of specialists can see patients?

Dr. Hofman: We moved into a brand-new facility in 2016. Our government invested A $\$ 1$ billion dollars to build a state-of-the art cancer center in Melbourne. Architecturally, it is a stunning and functional working environment. We have a dedicated area for outpatient theranostics within our cancer imaging department. This area is specially designed with lead-shielded glass surrounding patient cubicles, a bathroom for patients whose urine is radioactive, and a central area where patients can be monitored. We typically 
administer up to $8{ }^{177} \mathrm{Lu}$ treatments per day, currently 2 days per week. So, we have capacity to expand. We have a consultation room where we see new patients and follow up on patients after their restaging imaging. We have 7 full-time nuclear medicine physicians and a big team of technologists. As part of our growing prostate theranostics program, we have employed a genitourinary nurse specialist who is also doing a Masters to help work up the patients, review new referrals, and follow up with patients.

Dr. Calais: Who injects the patients with radiopharmaceuticals?

Dr. Hofman: It is usually our nuclear medicine technologists for imaging and nuclear medicine physicians for therapy. However, my own view is that once you are doing very high volumes that a nurse or nuclear medicine technologist should be the one giving the treatment under the supervision of a nuclear medicine specialist, similar to what happens in the chemotherapy day unit. I am confident that our nurses and technologists can deliver it as safely as (perhaps even more safely than) doctors.

Dr. Calais: Let us now look into the future of theranostics. What are your predictions for the future? What is the next major theranostic target?

Dr. Hofman: DOTATATE and PSMA theranostics have been remarkably successful. It is very rewarding to see patients' quality of life improve with these treatments. Now we have a lot of industry investment, and, over the next 5-10 years, we are going to see an acceleration of novel radiotracers for a variety of different malignancies. We are only at the beginning - despite the fact that this began 80 years ago with radioactive iodine for treating thyroid cancer. It strikes me that radioiodine is still the most effective theranostic we have in nuclear medicine. We use ${ }^{124}$ I PET/CT routinely in our thyroid cancer program, and we see $\mathrm{SUV}_{\max }$ results of more than 500 , even more than 2,500 in remnant tissue! It is therefore not surprising that radioactive iodine is highly effective. It's so remarkable that 80-year-old radioiodine has such incredible tumor targeting. Accordingly, we can find the next killer theranostic by looking for tracers that have extraordinary tumor-to-background contrast on PET images. Hoping for magic has caused many failures. Some new tumor-targeting theranostic agents in development have SUVs in the 10-15 range. With some exceptions, these are not likely to be killer theranostic compounds. Dosimetry is also a powerful tool when exploring new agents. Advances in radiochemistry combined with the many clinical radioisotopes available (including $\alpha$ and Auger electrons) indicate a bright future for theranostics.

Dr. Czernin: I think the trick is finding rational combination therapies. You may not need to achieve these extremely high target-tobackground ratios if your second therapeutic has synergistic effects.

Dr. Hofman: I completely agree, although the commercialization pathway makes it difficult to progress combinations for agents that don't show sufficient single-agent efficacy. In addition to combinations, we need to move these treatments earlier in the treatment paradigm. This has already been achieved for thyroid and neuroendocrine cancer, where theranostics is used as a first-line treatment. In prostate cancer, we have 5 theranostic clinical trials currently open at our center. Three are theranostic combinations with a programmed cell death protein-1 inhibitor (pembrolizumab), a poly-ADP-ribose polymerase inhibitor (olaparib), and an antiandrogen receptor-targeting agent (enzalutamide), respectively. And then we have 2 early-phase trials, one in men with newly diagnosed, high-volume castration-sensitive prostate cancer and one in men with high-risk localized prostate cancer. I hope to see a similar range of trials for a myriad different malignancies over the next few years.
And let's not forget that theranostics can also be useful beyond oncology, as evidenced by radioiodine for treating thyroid diseases.

Dr. Calais: Changing topics, you have an impressive presence on social media, particularly on Twitter. Do you find it useful, or is this just another distraction in our modern-day lives?

Dr. Hofman: I was introduced to hashtags by Declan G. Murphy, MD, at our genitourinary tumor board meetings. @declangmurphy bribed me with breakfast offers to start attending the 7:30 AM meeting. Back in 2014 this was already a core method of knowledge dissemination in urology. The COVID-19 pandemic has only accelerated the move away from paper journals and books. Young people no longer read the tables of contents but, instead, handpick the latest research to read from social media feeds, clicking to read articles that have generated the most interest. This really is a great way to keep up to date. Conference presentations and highlights are being posted live on Twitter, retweeted, and disseminated. It's a great way to connect and share information more quickly than ever before. In fewer than 140 characters, however, it's not the best way to deep dive or have nuanced debates.

Dr. Calais: To return to the beginning and the conclusion at the same time: How would you advise students thinking about a career in nuclear medicine?

Dr. Hofman: Nuclear medicine is one of the most dynamic and rapidly evolving specialties in medicine. My work balance of imaging, therapy, and research and seeing the benefit this has for patient outcomes makes most days inspiring and motivating. The patient problems I solve today are totally different from what I did in my first years of training. The technology is totally different. Every 2-3 years we have a new tracer, new equipment, and this keeps nuclear medicine a very exciting specialty to practice. Another exciting aspect of the job is the ability to collaborate with a wide variety of medical and nonmedical specialties, including radiopharmacy/radiochemistry, medical physics, computer science, and engineering. With this array of collaborations and technology, everyone can find something really interesting within nuclear medicine. In Australia, the job market is very positive for trainees from both radiology and internal medicine backgrounds. PET is the fastest growing area of radiology and extends beyond oncology, particularly within neurology and inflammatory or infectious diseases. Our ability to see what we treat with theranostics, seamlessly aligning imaging and therapy, has enormous potential to change paradigms in many diseases.

Dr. Czernin: Thank you, Michael, for taking the time to participate in this stimulating discussion.

\section{REFERENCES}

1. Hofman MS, Emmett L, Sandhu S, et al. [Lu]Lu-PSMA-617 versus cabazitaxel in patients with metastatic castration-resistant prostate cancer (TheraP): a randomised, open-label, phase 2 trial. Lancet. 2021;397:797-804.

2. Yamey G. John Violet: radiation oncologist and physician scientist who pioneered targeted radionuclide therapy for prostate cancer. BMJ. 2020;371:m4803

3. Hofman MS, Violet J, Hicks RJ, et al. $\left[{ }^{177} \mathrm{Lu}\right]$-PSMA-617 radionuclide treatment in patients with metastatic castration-resistant prostate cancer (LuPSMA trial): a single-centre, single-arm, phase 2 study. Lancet Oncol. 2018;19:825-833.

4. Violet J, Sandhu S, Iravani A, et al. Long-term follow-up and outcomes of retreatment in an expanded 50-patient single-center phase II prospective trial of Lu-PSMA-617 theranostics in metastatic castration-resistant prostate cancer. J Nucl Med. 2020;61: $857-865$.

5. Francis RJ, Bailey DL, Hofman MS, Scott AM. The Australasian Radiopharmaceutical Trials Network: clinical trials, evidence, and opportunity. J Nucl Med. 202162:755-756.

6. Hofman MS, Lawrentschuk N, Francis RJ, et al. Prostate-specific membrane antigen PET-CT in patients with high-risk prostate cancer before curative-intent surgery or radiotherapy (proPSMA): a prospective, randomised, multicentre study. Lancet. 2020;395:1208-1216. 